\title{
Management of pouch dysfunction in a tertiary centre
}

S Ourô, B Thava, I Shaikh and SK Clark.

Department of Surgery, St Mark's Hospital, Harrow HA1 3UJ, UK.

Corresponding author

Prof Sue Clark s.clark8@nhs.net 


\section{$\underline{\text { Abstract }}$}

Aim Restorative proctocolectomy (RPC) with ileal pouch anal anastomosis is the operation of choice for ulcerative colitis (UC) and some cases of familial adenomatous polyposis (FAP). Offering improvement in quality of life and high patient satisfaction, pouch surgery is also associated with significant morbidity. The aim of this study was to describe the management of patients referred to a tertiary centre with pouch dysfunction. Method All patients referred with pouch dysfunction from other institutions, between October 2006 and November 2014, were included in this retrospective study. Information regarding initial diagnosis prior to pouch procedure, type of procedure, symptoms leading to referral, relation of symptom appearance with ileostomy closure, investigations, final diagnosis, treatment and follow-up was reviewed.

Results One hundred and twenty one patients were included, with RPC mostly for UC (94\%), and with diverting ileostomy (83\%). The most frequent reasons for referral to our clinic were high defaecatory frequency in 83 (69\%) patients, abdominal pain and incontinence in 45 (37\%) each and perianal pain in 44 (36\%). The principal investigations performed were pouchoscopy in 97 (80\%) patients , examination under anaesthesia (EUA) in 62 (51\%), pelvic MRI in 56 (46\%) and pouchogram in $45(35 \%)$. The commonest diagnoses were pouchitis (primary and secondary) in 24 (21\%) patients and anastomotic leak in $26(22 \%)$. After full investigation a cause for the symptoms could not be found in $24(20 \%)$ patients, resulting in the diagnosis of exclusion of 'irritable pouch syndrome' or functional disorder .

Treatments given were long term antibiotic therapy in 29 patients $(25 \%)$, ileostomy in 19 (16\%), use of Medina catheter in $17(15 \%)$ and $12(10 \%)$ underwent dilatation under anaesthetic. Six patients $(5 \%)$ underwent revision pouch surgery with defunctioning ileostomy and another $6(5 \%)$ had pouch excision.

Conclusion Patients with ileoanal pouch dysfunction often have multiple symptoms. This study shows that a wide range of investigations and treatment modalities need to be available to manage such patients, with a specialised approach in a multidisciplinary setting. 


\section{'What does this paper add to the literature'}

Although associated with a low operative mortality, RPC has an associated morbidity as high as $30 \%$.

Most series addressing complications of RPC focus on management and outcome of particular pathologies. The aim of this study was to describe the overall approach to investigating patients with pouch dysfunction referred to a tertiary referral centre.

\section{$\underline{\text { Introduction }}$}

Restorative proctocolectomy (RPC) with ileal pouch anal anastomosis is a way of preserving intestinal continuity as an alternative to a permanent ileostomy and is the operation of choice for ulcerative colitis (UC) and some cases of familial adenomatous polyposis (FAP). Despite offering improvement in quality of life and high patient satisfaction, pouch surgery can be associated with a wide range of complications, both early and late.

Although associated with a low operative mortality, RPC has an associated morbidity as high as $30 \%{ }^{(1,2)}$. The most frequent complications include primary pouchitis, anastomotic leak and chronic peripouch sepsis. Other less frequently described complications associated with RPC include pouch vaginal, pouch perineal, pouch urinary or enterocutaneous fistulas, pouch inlet or outlet stenosis, previously unrecognised Crohn's disease, prepouch ileitis, prolapse, retained rectal mucosa and cuffitis, impaired anal sphincter function, small volume pouch, diversion pouchitis, bile salt malabsortion, 
irritable pouch syndrome, and pouch malignancy.

The overall pouch failure rate described in the literature is between 3 and $12 \%^{(1,2)}$. Various therapeutic options are available for the management of the various causes of pouch dysfunction. We have previously developed an algorithm ${ }^{(1)}$ to guide investigation and management of these challenging patients (Fig 1).

Most series addressing complications of RPC focus on management and outcome of particular pathologies (eg primary pouchitis, chronic sepsis), rather than the overall approach to a patient with pouch dysfunction. ${ }^{(3,4,5)}$

\section{$\underline{\text { Aims }}$}

The aim of this study was to describe the management of patients referred to a tertiary centre with pouch dysfunction.

\section{$\underline{\text { Methods }}$}

We performed a retrospective review of patients referred with pouch dysfunction from other institutions, first seen between October 2006 and November 2014. The information was taken from the patients' hospital records. Data collected included age, gender, initial diagnosis prior to pouch procedure (with or without covering ileostomy), symptoms leading to referral, relationship of symptom appearance with ileostomy closure, investigations, final diagnosis, treatment and follow-up.

\section{$\underline{\text { Results }}$}


One hundred and twenty one were included, 68 women (56\%) and 53 men (44\%), aged between 17 and 71 years (median 42 years). All had undergone RPC at another institution. The initial diagnosis prior to pouch procedure was UC in 112 patients (93\%), FAP in $7(6 \%)$ and indeterminate colitis (IC) in two (1\%). One hundred and four $(86 \%)$ patients initially had a covering ileostomy.

The most frequent reasons for referral to our clinic were high defaecatory frequency in 83 (69\%), patients, abdominal pain or incontinence in 45 (37\%) each, perianal pain in 44 (36\%), difficult evacuation in 33 (27\%), bleeding from the anus in $30(25 \%)$ or urgency in $24(20 \%)$. Other less frequent symptoms were also described (Table 1).

The majority of patients reported multiple (up to 9) symptoms (Fig 2).

In the group of patients who had a covering ileostomy at the time of original RPC (104 patients) symptoms of pouch dysfunction occurred within three months of stoma closure in $52(50 \%)$. In those who had initial acceptable pouch function for 3 months after stoma closure, symptoms commenced later, between 3 and 345 months after ileostomy closure, with a median time to appearance of 39 months. In the group of patients who had not had a covering stoma (13 patients) symptoms of pouch dysfunction started between 1 and 216 months (median 1 month) from the initial pouch operation.

The most frequent investigations performed at our institution were pouchoscopy in 97 $(80 \%)$ patients, examination under anaesthesia (EUA) in $62(51 \%)$, pelvic MRI in 56 (46\%), pouchogram in $45(37 \%)$ and barium follow through in 33 (27\%) (Table 2).

We found that most patients had more than one final diagnosis. The most frequently encountered were pouchitis (primary and secondary) in $24(20 \%)$ patients and anastomotic leak in $26(22 \%)$. After full investigation a cause for the symptoms could not be found in $24(20 \%)$ patients, resulting in the diagnosis of exclusion of 'irritable pouch syndrome' or functional disorder. 
In the anastomotic leak group, $24(92 \%)$ of the leaks were found in the pouch-anal anastomosis and two (8\%) in the blind end of the pouch. We identified $12(10 \%)$ patients with chronic peripouch sepsis, 10 caused by anastomotic leaks already included in the former group and two without an identifiable leak. Fourteen (12\%) patients had a pouchvaginal fistula, four from the anastomosis, again already included in the anastomotic leak group. Pouch outlet stenosis was found in $15(12 \%)$ patients and significant retained rectum with and without cuffitis in $10(8 \%)$ each.

Two patients were found to have previously unrecognised malignancies causing outlet stenosis. Other less frequent diagnoses were also identified (Table 3). Most patients had more than one diagnosis.

Seventy five patients $(62 \%)$ were referred purely with symptoms but $42(37 \%)$ were referred with a diagnosis that was confirmed in 21 (50\%). In 21 patients (50\%) the final diagnosis was different.

Concerning treatment, the most frequent was long term antibiotic therapy in 29 patients (24\%), ileostomy in $19(16 \%)$, use of Medina catheter in $17(14 \%)$ and $12(10 \%)$ underwent dilatation under anaesthetic. Six patients $(5 \%)$ underwent revision pouch surgery with defunctioning ileostomy and another 6 had pouch excision (5\%). Other treatments included seton placement in $8(7 \%)$, prescription of bile salt sequestrants in 8 (7\%) and drainage of peripouch sepsis in five (4\%), oral immunosupressants and biofeedback in three each (3\%), enemas and suppositories (anti-inflammatories or alicaforsen), barrier creams, botulinum toxin injection and advancement flap for fistula in two each $(2 \%)$ and diltiazem ointment, enterocutaneous fistula repair and fistulotomy in one patient each (1\%). Two patients are awaiting pouch excision following neoadjuvant chemotherapy and defunctioning stoma (Table 4).

With regard to follow-up, $18(15 \%)$ patients had only one appointment in our department. Ten of those chose to continue follow-up in their referring hospital, three did not attend 
the scheduled follow-up appointment and three declined the proposed surgical treatment and were discharged. Two were discharged after one attendance only. Ninety nine $(82 \%)$ other patients have been followed-up at our institution.

\section{$\underline{\text { Discussion }}$}

Although associated with an improvement in quality of life, RPC can result in significant morbidity. Symptom appearance can be almost immediate, happening in the first three months after ileostomy closure, but can also be delayed and appear many years later. In our cohort, some patients had a very late onset of symptoms, appearing at a median time of 39 months and up to 345 months after ileostomy closure.

There are many symptoms associated with pouch dysfunction and the majority of patients present with several. Due to the overlap of symptoms and complexity of the problems resulting in pouch dysfunction, a number of investigations may be necessary to reach a diagnosis. Likewise, a wide range of heterogeneous pathologies can be associated with pouch dysfunction.

It is interesting to note that, in our series, $50 \%$ of those given an initial diagnosis of the cause of their symptoms had that diagnosis changed after full investigation. This reflects the need for a specialised approach with the use of an algorithm of management ${ }^{(1)}$. In this study, $20 \%$ of patients remained with the diagnosis of exclusion of 'functional pouch disorder' despite full investigation. We found two cases of unrecognized adenocarcinoma of the pouch outlet and no histologically confirmed cases of Crohn's disease. A wide variety of treatments were employed, ranging from simple pouch evacuation techniques to pouch revision or excision.

This study has a number of weaknesses, particularly referral bias, as many straightforward problems will have been successfully managed at patients' local hospitals, so tertiary referral will not have been required. No meaningful data can be 
gleaned on the effectiveness of various management strategies, as the symptoms and pathologies identified were so numerous that the number of each is very low.

This series does, however, show the diversity involved in managing pouch dysfunction patients in a tertiary referral centre. A wide range of investigations and treatment modalities need to be available to manage such patients effectively. This is best done in a multidisciplinary setting by a team experienced in managing pouch dysfunction, including surgeons, gastroenterologists, endoscopists, radiologists and pathologists. Given the relatively low numbers of RPC's performed, and the even lower frequency of subsequent problems, it would seem logical that such cases are managed in specialist units. 
Fig 1 Algorithm for the investigation of pouch dysfunction

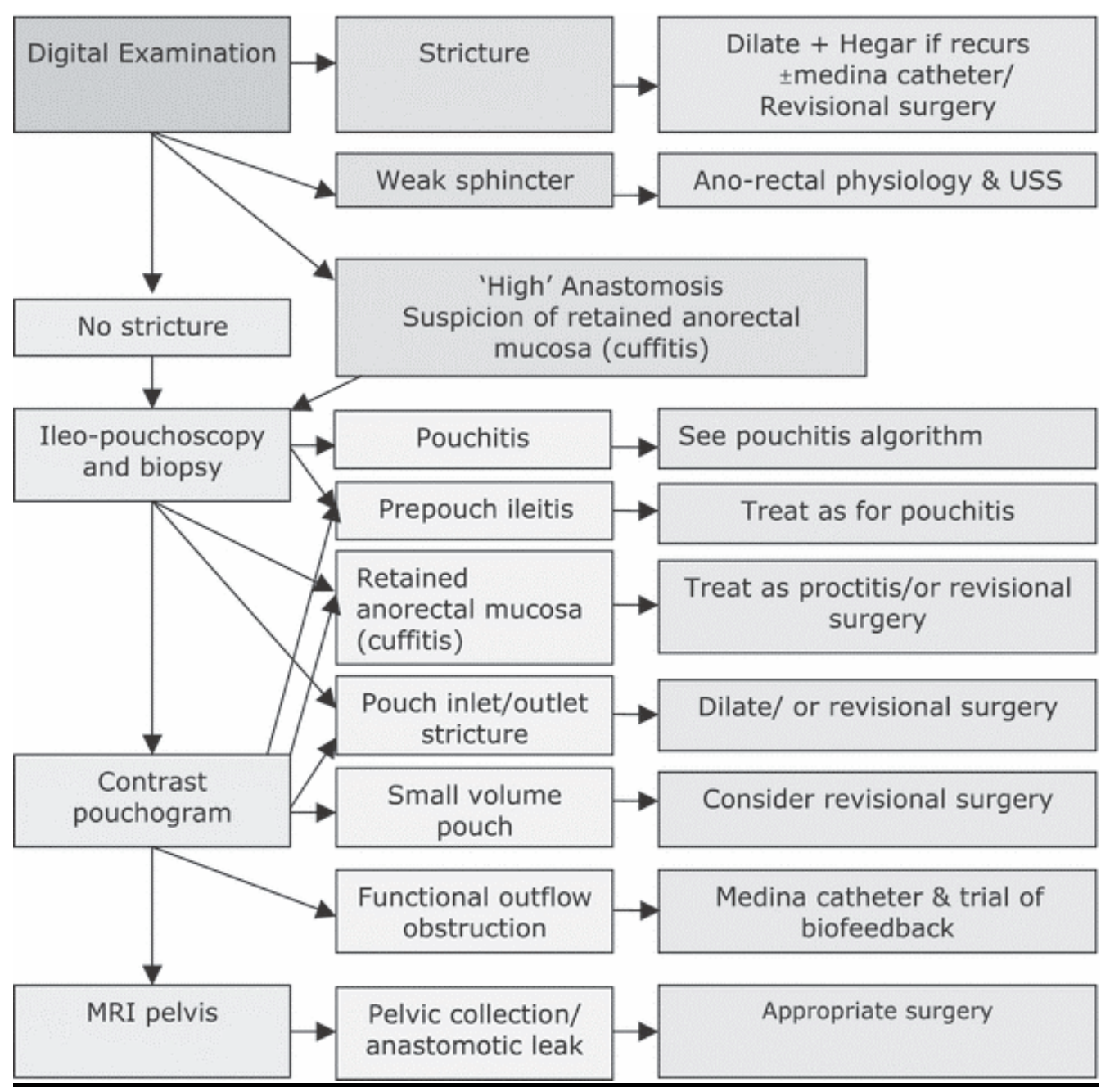

Reproduced fromMcLaughlin SD, Clark SK, Tekkis PP et al; Review article: restorative proctocolectomy, indications, management of complications and follow-up--a guide for gastroenterologists; Aliment Pharmacol Ther. 2008 May;27(10):895-909 with permission 
Fig 2 Number of symptoms at presentation

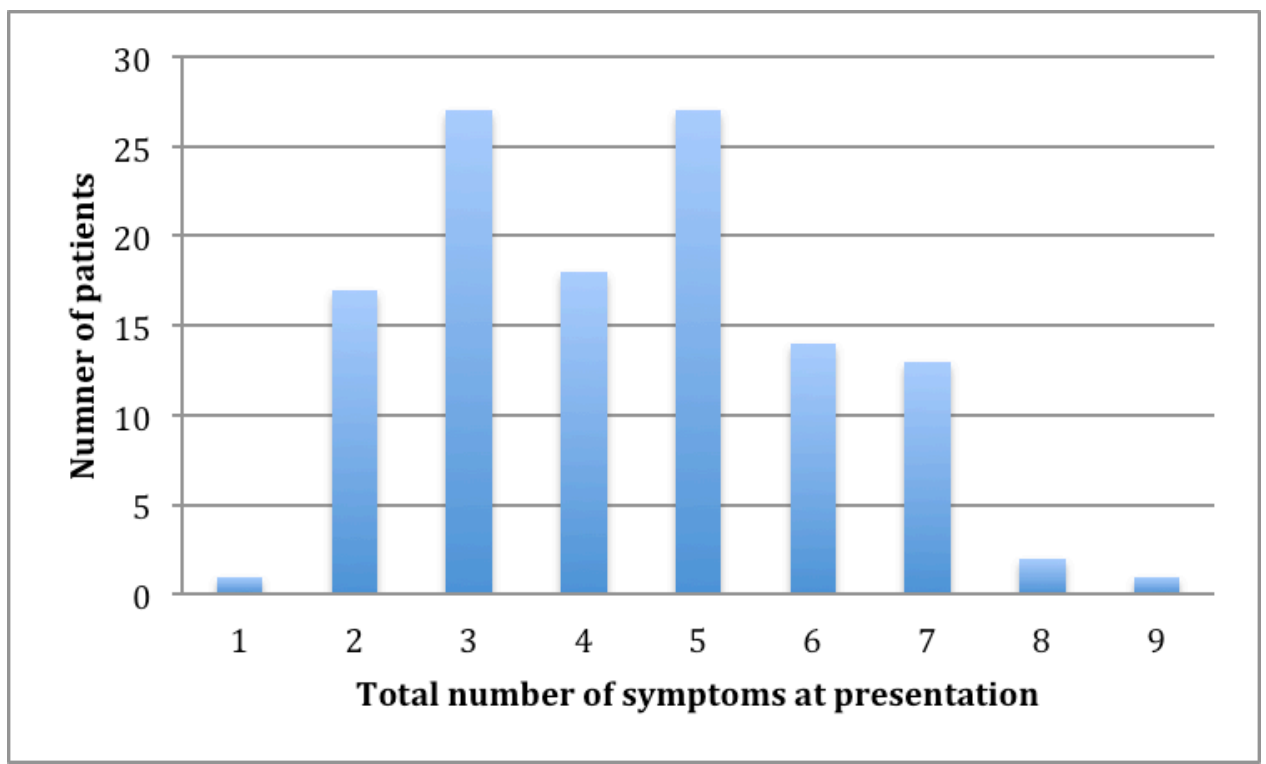


Table 1 Symptoms leading to referral

\begin{tabular}{|l|l|}
\hline Symptom & N (\%) \\
\hline High defaecatory frequency & $83(69)$ \\
\hline Abdominal pain & $45(37)$ \\
\hline Incontinence & $45(37)$ \\
\hline Perianal pain & $44(36)$ \\
\hline Difficult evacuation & $33(27)$ \\
\hline Bleeding from the anus & $30(25)$ \\
\hline Urgency & $24(20)$ \\
\hline Watery faeces & $22(18)$ \\
\hline Mucous anal discharge & $18(15)$ \\
\hline Faecal vaginal discharge & $17(14)$ \\
\hline Purulent anal discharge & $8(7)$ \\
\hline Vomiting & $8(7)$ \\
\hline Enterocutaneous fistula & $6(5)$ \\
\hline Purulent perianal discharge & $5(4)$ \\
\hline Abdominal distension & $5(4)$ \\
\hline Pouch prolapse & $3(3)$ \\
\hline Weight loss & $3(3)$ \\
\hline Pneumaturia/ fecaluria & $2(2)$ \\
\hline
\end{tabular}


$\underline{\text { Table } 2}$ Investigations performed

\begin{tabular}{|l|l|}
\hline Investigations & N (\%) \\
\hline Flexible pouchoscopy & $97(80)$ \\
\hline EUA & $62(51)$ \\
\hline MRI pelvis & $56(46)$ \\
\hline Pouchogram & $45(37)$ \\
\hline Barium follow through & $33(27)$ \\
\hline CT abdomen and pelvis & $20(17)$ \\
\hline Anal physiology & $14(12)$ \\
\hline MRI enterography & $13(11)$ \\
\hline Endoanal ultrasound & $9(7)$ \\
\hline Defecating pouchogram & $8(7)$ \\
\hline SeHCAT test & $6(5)$ \\
\hline Diagnostic laparoscopy & $4(3)$ \\
\hline Fistulogram & $3(3)$ \\
\hline
\end{tabular}


Table 3 Final diagnosis

\begin{tabular}{|l|l|}
\hline Final diagnosis & n (\%) \\
\hline Anastomotic leak & $26(21)$ \\
\hline Functional disorder & $24(20)$ \\
\hline Primary idiopathic pouchitis & $24(20)$ \\
\hline Pouch outlet stenosis & $15(12)$ \\
\hline Pouch-vaginal fistula & $14(12)$ \\
\hline Chronic peripouch sepsis & $12(10)$ \\
\hline Cuffitis & $10(8)$ \\
\hline Bile salt malabsortion & $8(7)$ \\
\hline Retained rectum without inflammation & $7(6)$ \\
\hline Impaired anal sphincter function & $6(5)$ \\
\hline Anal fissure & $6(5)$ \\
\hline Prepouch ileitis (without pouchitis) & $4(3)$ \\
\hline Fistula in ano & $4(3)$ \\
\hline Entrocutaneous fistula & $4(3)$ \\
\hline Pouch inlet stenosis & $3(3)$ \\
\hline Small bowel obstruction & $2(2)$ \\
\hline Pouch prolapse / solitary ulcer & $2(2)$ \\
\hline Small pouch & $1(1)$ \\
\hline Diversion pouchitis & $1(1)$ \\
\hline Pouch-urethral fistula & $1(1)$ \\
\hline
\end{tabular}


Table 4 Treatment

\begin{tabular}{|l|l|}
\hline Treatment & N (\%) \\
\hline Long term antibiotics & $29(24)$ \\
\hline Defunctioning ileostomy & $19(16)$ \\
\hline Medina catheterisation & $17(14)$ \\
\hline Dilation under general anaesthesia & $12(10)$ \\
\hline Seton placement & $8(7)$ \\
\hline Bile sequestrants & $8(7)$ \\
\hline Redo pouch & $6(5)$ \\
\hline Pouch excision & $6(5)$ \\
\hline Drainage of peripouch sepsis & $5(4)$ \\
\hline Immunosupressors (oral) & $3(3)$ \\
\hline Biofeedback & $3(3)$ \\
\hline Dietary change advised & $2(2)$ \\
\hline Anti-inflammatory / alicaforsen enema & $2(2)$ \\
\hline Barrier creams & $2(2)$ \\
\hline Botox injection of sphincter & $2(2)$ \\
\hline Advancement flap & $2(2)$ \\
\hline $\begin{array}{l}\text { Defunctioning and neoadjuvant therapy for } \\
\text { malignancy (pouch excision pending) }\end{array}$ & $2(2)$ \\
\hline Diltiazem (topical) & $1(1)$ \\
\hline Enterocutaneous fistula repair & $1(1)$ \\
\hline Fistulotomy & $1(1)$ \\
\hline
\end{tabular}




\section{$\underline{\text { References }}$}

(1) McLaughlin SD, Clark SK, Tekkis PP (2008) Review article: restorative proctocolectomy, indications, management of complications and followup--a guide for gastroenterologists; Aliment Pharmacol Ther. May;27(10):895-909

(2) Fazio VW, Kiran RP, Remzi FH (2013) Ileal pouch anal anastomosis: analysis of outcome and quality of life in 3707 patients; Ann Surg. Apr;257(4):679-85.

(3) Lichtenstein GR, Cohen R, Yamashita B (2006) Quality of life after proctocolectomy with ileoanal anastomosis for patients with ulcerative colitis; J Clin Gastroenterol. Sep;40(8):669-77.

(4) Fazio VW, Kiran RP, Remzi FH et al (2013)Ileal pouch anal anastomosis: analysis of outcome and quality of life in 3707 patients; Ann Surg. Apr;257(4):679-85

(5) Shen B, Remzi FH, Lavery IC, Lashner BA, Fazio VW (2008) A proposed classification of ileal pouch disorders and associated complications after restorative proctocolectomy; Clin Gastroenterol Hepatol. Feb;6(2):145-58 